\title{
Increasing Effectiveness of Model-Based Fault Diagnosis: A Dynamic design for Decision Making
}

\author{
C. ANGELI and A. CHATZINIKOLAOU
}

\begin{abstract}
A large diversity of advanced methods for automated fault detection has been developed with a variety of techniques in different domains. The effectiveness of the diagnosis for technical processes is highly improved when the diagnostic system is able to conclude in parallel to the dynamic industrial processes. This paper presents the design a diagnostic approach which aims to increase the performance of the decision making in fault diagnosis. The proposed approach takes into account the knowledge form the reliability analysis and the model-based fault dynamic component behavior. The effectiveness and the performance of the technique are illustrated by a case study.
\end{abstract}

Keywords - Modelling, Simulation, Fault diagnosis, Dynamic Process.

\section{Introduction}

High reliability, efficiency and safety of many industrial processes require the development of effective fault detection and diagnosis methods. Faults in systems are usually diagnosed using analytical redundancy by comparing the measured and the corresponding estimated outputs of the system.

\section{ANGELI}

Piraeus University of Applied Sciences

Laboratory of Applied Mathematics and Artificial Intelligence

P. Ralli \& Thivon 250

GR-17121 Athens, Greece

\section{A. CHATZINIKOLAOU}

Laboratory of Applied Mathematics and Artificial Intelligence

S. Patsi 62

GR-118 55 Athens, Greece
A short historical view of fault detection and isolation can also be found in [1], [2] and current developments can be observed in [3], [4], [5]. The diagnostic procedure is composed of three stages: residual generation, residual evaluation and finally decision making.

In principle, decision making is realized according to the elementary logic. Some specific mathematic algorithms can improve the efficiency of the decision making as [6], [7], [8]. However the binary data produced by residual evaluation are poor in information and as consequence some other knowledge to the residuals can be considered for fault isolation. Researchers [9], [10], [11], [12], [13], [14] have been proposed that it can be useful to combine qualitative and quantitative knowledge to improve the fault diagnosis efficiently. Fault isolation performance can increase through the integration of other source of knowledge in the diagnostic procedure.

The aim of this paper is to propose a new approach in order to increase the performance of the decision making in fault diagnosis taking into account a priori knowledge of the system state of the dynamic performance.

The paper is organized as follows: Section 2 presents the evolution of intelligent fault detection techniques. Section III focuses on the modelling, simulation and model validation process, while Section IV presents the decision making design and the justification of the results. Finally, Section $\mathrm{V}$ includes concluding remarks. 


\section{Evolution of intelligent Fault techniques}

On-line fault detection techniques have been developed for automated processes during the last few years. These methods include numerical methods, artificial intelligence methods or combinations of the two methodologies. Artificial intelligence methods allow the development of effective diagnostic applications especially in the case of very complex timevarying and non-linear systems.

Neural networks find application in fault detection due to their main ability of pattern recognition. The network is trained to learn, from the presentation of the examples, to form an internal representation of the problem. For on-line fault detection it is needed to relate the sensor measurements to the causes of faults, and distinguish between normal and abnormal states. A number of input signals have to be weighted, added and combined to form output signals. It is necessary to cascade several layers in order to display complex relationships. The weighting factors are determined interactively on the basis of training data containing both the input signals and the corresponding output signals. Training is completed once the deviation drops below the prescribed permitted value. After training the generated weighting matrix contains all of the information required to conclude the fault status of the controlled system from a new measured step response on the basis of the reduced number of interpolation points.

Qualitative models of normal and faulty equipment are simulated to describe the range of possible behaviours of the operation of a system without numeric models. The modelling of physical situations contains a set of qualitative equations derived from a set of quantitative equations or from qualitative descriptions about relationships among the process variables and contains knowledge about structure, function and behaviour. Sensor data from actual processes are used to select between the different developed models. The fault diagnosis is performed by comparing the predicted and observed behaviour of a system.

Expert systems are find applications in the area of fault diagnosis from their early stages. In model-based expert systems expert knowledge is contained primarily in a model of the expert domain. These systems offer more robustness in diagnosis because they can deal with unexpected cases that are not covered by the classical heuristic applications. The knowledge bases of such systems are less expensive to be developed because they do not require field experience for their building. In addition, they are more flexible in case of design changes. Model-based diagnosis systems offer flexibility and accuracy but they are also domain dependent.

\section{Modelling and Simulation}

The modelling of the systems elements leads to a non-linear system of equations. This system can be written in the following state vector form:

$\overline{\dot{x}}=f(\bar{x}) \quad$ where $\quad \bar{x}=\left[\begin{array}{c}p_{a} \\ p_{b} \\ \dot{\varphi} \\ \varphi\end{array}\right]$

Thus:

$$
\left[\begin{array}{c}
\dot{p}_{a} \\
\dot{p}_{b} \\
\ddot{\varphi} \\
\dot{\varphi}
\end{array}\right]=f\left[\begin{array}{c}
p_{a} \\
p_{b} \\
\dot{\varphi} \\
\varphi
\end{array}\right]
$$

The results of the simulation process for the pressures $p_{a}$ and $p_{b}$ at the ports of the hydraulic motor are presented in Figure 1. 

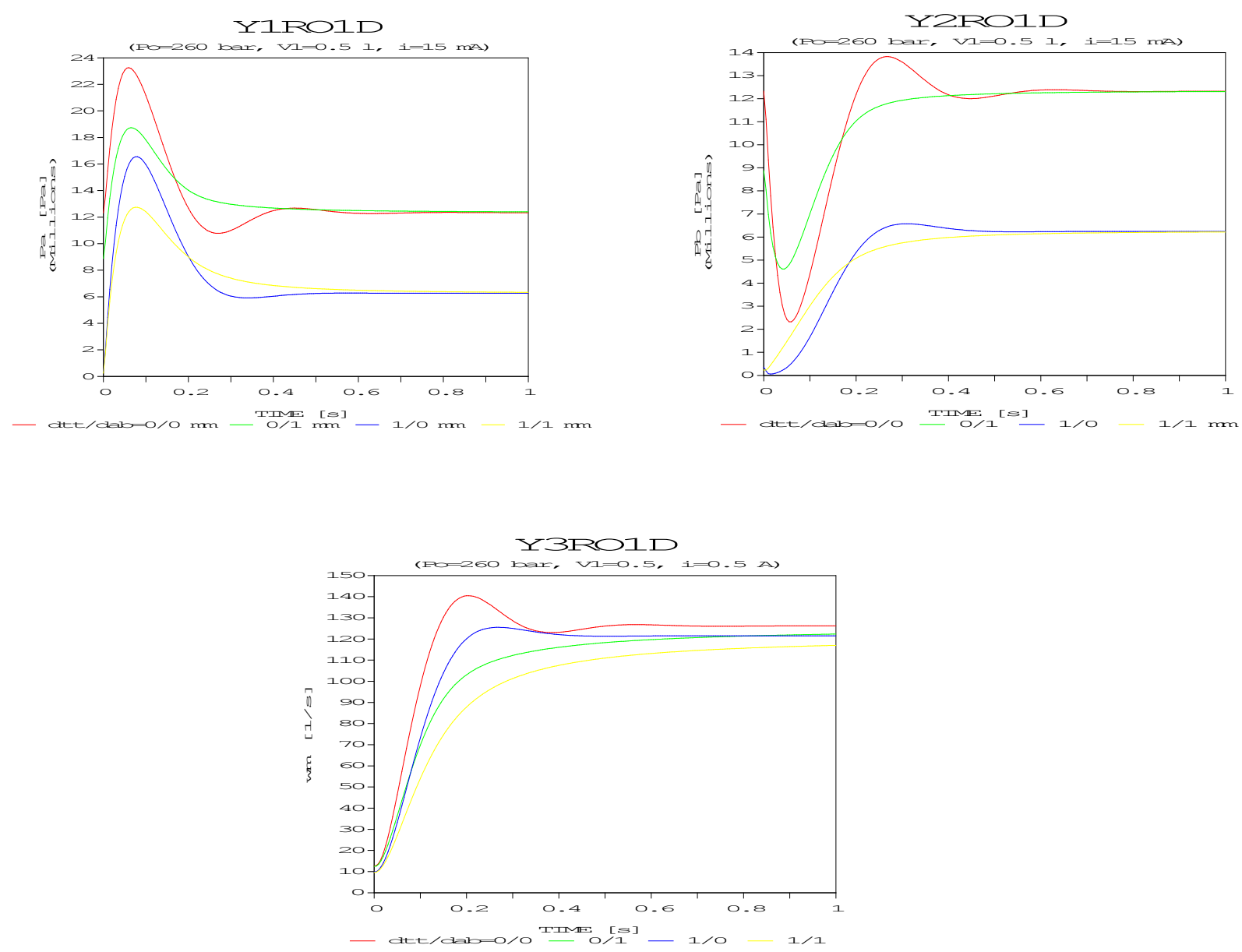

Figure 1. Simulation results

The simulation results were validated after a comparison with the actual measurements.

In order to validate the simulation model, the actual system was connected through sensors with a data acquisition system and the relevant curves of the variables were produced. The acquired data were compared with the simulation results. The sensor measurements refer to the pressures at critical points of the hydraulic system and the angular velocity. The objective of the validation process was mainly the estimation of the uncertain parameter values, as for example the parameter value of the friction torque, so that the model's output behaviour has a satisfactory accuracy when compared with the behaviour of the actual system under various operating conditions using various values for the bypass orifices.

Values from the simulation and from measurements are illustrated in Figure 2. Under consideration of the comparison results it can be concluded that the mathematical model is realistic and reasonably precise since the experimental results are consistent with the simulation results. 


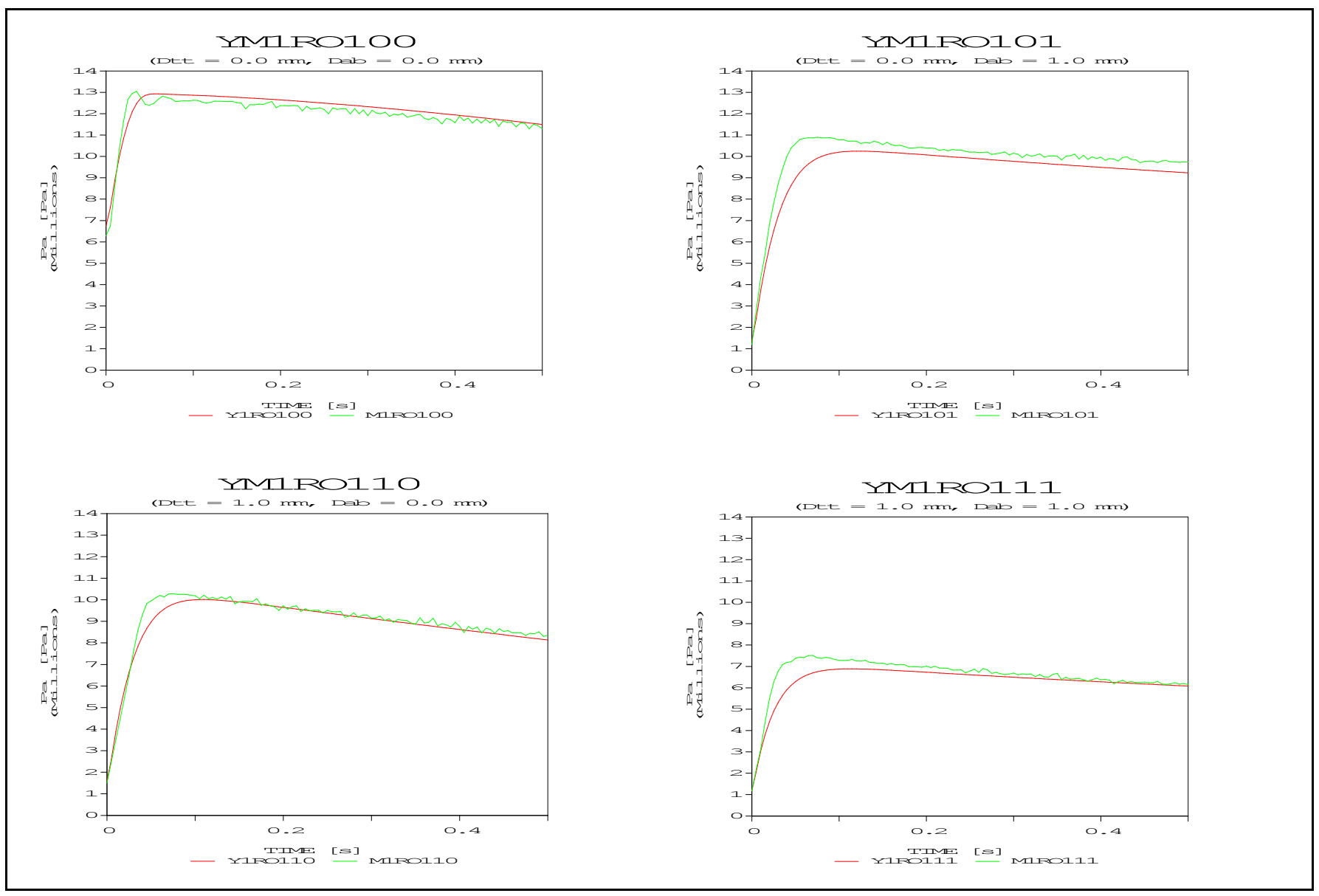

Figure 2. Comparison of measured and calculated results.

\section{Decision making design}

Faults are detected by setting a threshold on a residual generated from the difference between measurements and estimates of these measurements. The residual signals carry information on the time and isolation of the faults. When a fault occurs, the deviation from zero is such that the new condition can be distinguished from the fault free working mode.

The role of the decision system is to determine whether the residuals differ significantly from zero and to decide about the faulty component. Figure 3 presents a diagram of the diagnosis process using system's models.
This system requires a knowledge base architecture that permits the interaction of sensor information, modelling information and experiential knowledge in symbolic representation. Experiential knowledge was complementary used to the scientific knowledge of the mathematical model in order to model more precisely the expert's reasoning activity, to gain the efficiency of heuristics and the advantages of a real world application.

The interaction of the various sources of information and knowledge was realized by knowledge representation scheme the "topic" that offers the opportunity to read external linguistic information from files that could be combined with the stored knowledge. 
Proc. of the Sixth International Conference on Advances in Computing, Electronics and Communication - ACEC 2017.

Copyright $(\odot$ Institute of Research Engineers and Doctors. All rights reserved.

ISBN: 978-1-63248-138-2 doi: 10.15224/ 978-1-63248-138-2-18

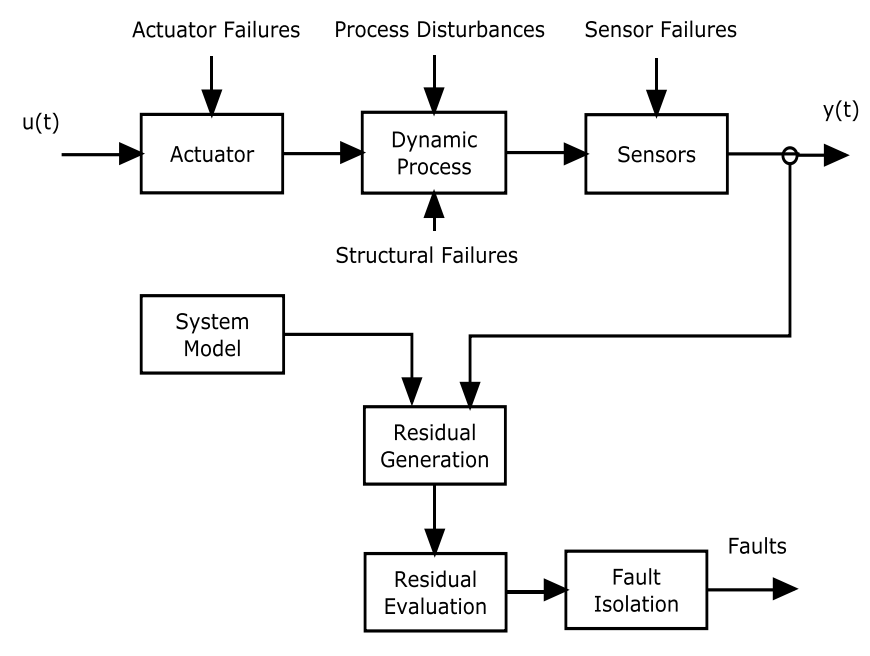

Figure 3. The fault detection design

The system was tested for various cases of malfunction using simulated faulty conditions to examine the performance of system and to validate the presented method. It was found that a variation $\pm 5 \%$ of the system parameters that include uncertainty does not affect the effectiveness of the fault detection process.

\section{Conclusion}

The increasing complexity of industrial systems leads to increased requirements concerning diagnosis capabilities. The diagnostic approach presented in this paper increases the performance of the decision making in model based fault diagnosis. The developed approach takes into account a priori knowledge of the system state and knowledge of the dynamic performance while combines effectively scientific with experiential knowledge. The research identifies new opportunities to further development of automated tasks for these systems and as consequence a higher degree of reliability of the operation of these systems. The simulation example highlights the performance of the method.

\section{References}

[1] R. Isermann and P. Balle, Trends in the Application of Model Based Fault Detection and Diagnosis of Technical Processes. IFAC World Congress 1996, San Francisco.

[2] P.M Frank., Analytical and qualitative model-based fault diagnosis: A survey and some new results. European Journal of control, Vol. 2, 1996, pp 6-28.

[3] R. Patton, P. Frank and R. Clark, Issues in Fault Diagnosis For Dynamic Systems, Springer-Verlag, 2000.

[4] R. Isermann, Model-based Fault Detection and Diagnosis: Status and Applications. Annual Reviews in Control, 29, 2005, pp. 71-85.

[5] J. Korbicz, Koscielny J., Kowalczuk Z. and Cholewa W., Fault Diagnosis: Models, Artificial Intelligence, Applications, 2004, Springer Verlag, Berlin.

[6] J. Chen and R. Patton, Robust Model Based Fault Diagnosis For Dynamic Systems, Kluwer Academic Publishers, 1999.

[7] S. Narasimhan and G. Biswas, Model-based diagnosis of hybrid systems, IEEE Transactions on Systems, Man and Cybernetics, A Syst. Humans, Vol. 37, No. 3, 2007, pp. 348-361.

[8] R. Isermann, Fault diagnosis applications: Model-based condition monitoring: Actuators, Drives, Machinery, Plants, Sensors and Fault tolerant Systems, Springer, Berlin, 2011.

[9] C. Angeli, On-line Expert Systems for Fault Diagnosis in Technical Processes. Expert Systems, Vol. 25, No2, 2008, pp. 115-132.

[10] M. Nyberg and M. Krysander, Combining AI, FDI and Statistical Hypothesis-Testing in a Framework for Diagnosis, In Proceedings Safeprocess 03, Washington, U.S.A., 2003, pp. 813-818

[11] I. Hwang, S. Kim and Y. Kim, A Survey of fault Detection, Isolation and Reconfiguration Methods, IEEE Transactions on Control Systems Technology, 2009.

[12] Su Hua and Chong Kil-To, Neural network based expert system for Induction Motor Faults Detection, Journal of Mechanical Science and Technology, Vol. 20 (7), 2006, pp. 929-940.

[13] Y. Kun, O-Y. Guangyao and Y. Lina, Research upon Fault Diagnosis Expert System Based on Fuzzy Neural Network," icie, vol. 1, pp.410-413, 2009 WASE International Conference on Information Engineering, Shanxi, China, 2009, July 10-July 11.

[14] C.Angeli and A. Chatzinikolaou, On-Line Fault Detection Techniques for Technical Systems: A Survey. International Journal of Computer Science \& Applications, Vol. 1, No. 1, 2004, pp. 12-30. 